\title{
Multi-Pulse Diode Rectifier for More-Electric Aircraft Applications: Parallel versus Series Topologies
}

Ahmed A. A. Hafez ${ }^{1,2}$

\author{
${ }^{1}$ Electrical Engineering Department, Faculty of Engineering, Assiut University, Egypt \\ ${ }^{2}$ Electrical Engineering Department, College of Engineering, Shaqra University, KSA, \\ aabdelhafez@su.edu.sa
}

\begin{abstract}
This article analyzes thoroughly the performance of the Multi-Pulse Diode Rectifiers (MPDRs) regarding the quality of input/output voltage and currents. Two possible arrangements of MPDRs are investigated: series and parallel. The impact of the DC side connection on the performance of the MPDRs regarding the operation parameters and rectifier indices are comprehensively examined. Detailed analytical formulas are advised to identify clearly the key variables that control the operation of MPDRs. Moreover, comprehensive simulation results are presented to quantify the performance and validate the analytical analysis. Test-rig is set up to recognize the promising arrangement of MPDRs. Significant correlation is there between simulation and practical results. The analytical results are presented for aircraft systems $(400 \mathrm{~Hz})$, and power grid systems $(60 \mathrm{~Hz})$. This is to study the impact of voltage and frequency levels on the topology type of MPDRs. In general, each topology shows merits and have limitations.
\end{abstract}

Keywords : Multi-Pulse Diode Rectifier, More-Electric Aircraft, DC Distribution System, Parallel, Series, Total Harmonic Distortion.

\section{Introduction}

More-Electric Aircraft (MEA) approach investigates transmitting and distributing the on-board electric energy via a DC distribution system. This approach offers significant reduction in the weight, the size and the losses, while increasing the levels of the transmitted power and improving the system regulation [1-3].

MPDRs are the promising candidate for implementing the front end converters for such DC distribution system [4,5]. They produce the required DC voltage level with minimal ripple content. Moreover, the MPDRs have reduced volumetric dimension/weight, evaluated efficiency and reduced Electro-Magnetic Interference (EMI). Furthermore, they are simple and inexpensive [69]. The MPDRs particularly with pulses more than twelve has the merit of complying with restrict harmonic standards [10].

This article addresses the impact of DC side connection of MPDRs on the performance regarding the quality of input/output voltage and currents. The contributions of this article are :

i. Advising analytical formulas for input/output voltage and current in MPDRs either parallel or series topologies.

ii. Analyzing the waveforms of input/output current and voltage regarding ripple and harmonic levels.

iii. Identifying the merits and drawback of each operation mode of MPDRs.

\section{Multi-Pulse Diode Rectifier}

Six-pulse diode rectifier is the main building block for the MPDRs, Figure 1. Thus, MPDRs have two main topologies according to the connection of the output sides of the six-pulse diode rectifier, Figure 1. They are series and parallel connected MPDRs.

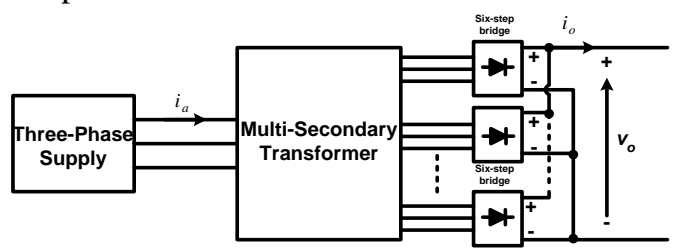

(a)

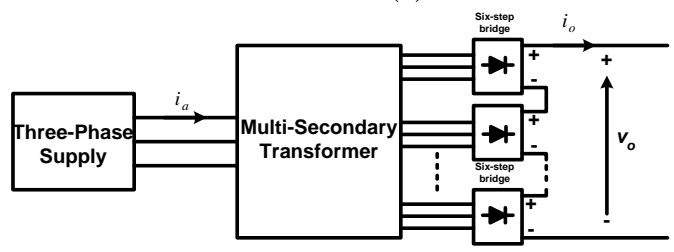

(b)

Figure 1 : MPDRs (a) Parallel connected (b) Series connected

The input sides of six-pulse diode rectifier are connected to multi secondary windings of a transformer with appropriate phase shift, $\theta$. This phase shift, $\theta$, is related to number of pulses on the DC side of the MPDRs by,

$$
\theta=\frac{360^{\circ}}{m}
$$

where $m$ is number of pulses, $m$ is multiple of 6 . 


\section{A: Output Side of MPDR}

The output voltage of MPDR is given by (2). It has two components. One is a DC component, $\mathrm{V}_{\mathrm{dc}}$, that represents the effective load voltage, and it obtained by averaging the output voltage over one cycle of the supply. The other is a ripple component that has $\mathrm{m}$ times the frequency of the supply.

The value of $\mathrm{V}_{\mathrm{dc}}$ varies between series and parallel topologies. Equation (3) gives the average DC voltage, $\mathrm{V}_{\mathrm{dc}}$ for series and parallel topologies.

$$
v_{o}=\mathrm{V}_{\mathrm{dc}}-\sum_{n=m, 2 m}^{\infty} \frac{2 \hat{\mathrm{V}}_{m}}{\left(n^{2}-1\right)} \frac{m}{\pi} \sin \left(\frac{\pi}{m}\right) \cos \left(\frac{n \pi}{m}\right) \cos (n \omega t)
$$

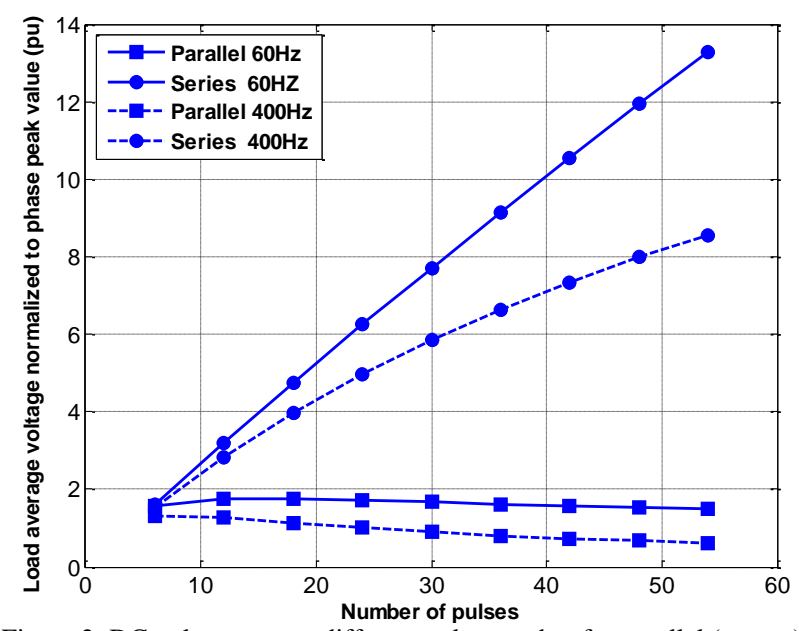

Figure 2: DC voltage versus different pulse number for parallel (square) and series (circle), solid $(60 \mathrm{~Hz})$, dashed $(400 \mathrm{~Hz})$

$$
\mathrm{V}_{\mathrm{dc}}=\left\{\begin{array}{lc}
\sin \left(\frac{2 \pi}{m}\right) \hat{\mathrm{V}}_{m}\left[\frac{m}{\pi}+R_{o n}\left(\frac{m}{2 \pi}\right) \frac{-\gamma+\sin \gamma}{\omega L_{c}}\right]-\left[\frac{m}{2 \pi} \omega L_{c}+R_{o n}\left(2-\frac{m \gamma}{\pi}\right)\right] I_{d c}-2 V_{o n} & \text { Parallel } \\
\frac{m \sqrt{3}}{2 \pi} \hat{\mathrm{V}}_{m}-\left[\frac{m}{2 \pi} \omega L_{c}+\frac{m}{3} R_{o n}\right] I_{d c}-\frac{m}{3} V_{o n} & \text { Series }
\end{array}\right.
$$

where $\hat{V}_{m}$ is the peak of phase voltage, $L_{c}$ is the supply inductance; $\gamma$ is commutation angle. $\mathrm{I}_{\mathrm{dc}}$ is the average DC current. $\mathrm{R}_{\text {on }}$ and $\mathrm{V}_{\text {on }}$ are on-state resistance and voltage drop of diode during on-state.

The commutation angle $\gamma$ is given by,

$$
\gamma=\cos ^{-1}\left(1-\frac{\omega L_{c} I_{d c}}{\hat{\mathrm{V}}_{m} \sin \left(\frac{2 \pi}{m}\right)}\right)
$$

The average value of output voltages, $\mathrm{V}_{\mathrm{dc}}$, of MPDRs from (3) for different number of pulses are shown in Figure 2. The DC voltage $\mathrm{V}_{\mathrm{dc}}$ is normalized with respect to the peak value of supply phase voltage, $\hat{\mathrm{V}}_{m}$. This is to generalize the analysis for any AC supply with avaraible amplitude. However, Two different set of data are used for producing Figure 2. One set for aircart $400 \mathrm{~Hz}$ supply, while the other is for $60 \mathrm{~Hz}$ grid power supply. In producing Figure 2, the diode is modeled during the onstate by a resistance $R_{\text {on }}$ of $1 \mathrm{~m} \Omega$, a forward voltage $V_{\text {on }}$ of $0.8 \mathrm{~V}$. The commutation inductance $\mathrm{L}_{\mathrm{c}}$ is set to $1 \mathrm{mH}$ in Figure 2.

The series topology produces significant high value of the DC voltage, which is crucial for applications requiring high DC voltage as adjustable speed motor drives [6, 7]. Figure 2 shows that DC voltage of the parallel connection decreases as the pulse number increases. This is attributed to the increasing number of conducting diodes with pulse number. Therefore, more voltage drop results from the operation of high pulse parallel MPDRs.

Figure 2 shows that DC voltage decreases as the frequency increases, particularly for series topology. As, the series topology for $60 \mathrm{~Hz}$ produces around $34 \%$ increase in the output voltage than the operation at $400 \mathrm{~Hz}$ at pulse number of 30 . The impact of frequency is insignificant for MPDR parallel topology, Figure 2.

Figure 2 indicates that series topology is preferred option for applications requiring very high level of DC voltage. Meanwhile, parallel topology suits high power application reasonably.

Equation (2) shows that the ripples in the output voltage is composed of infinite number of harmonics, which their frequencies are multiple of pulse number. The amplitudes of these harmonics are $(1 / \mathrm{k})^{2}$ times the amplitude of the first harmonic, where $\mathrm{k}$ is equal to $\mathrm{m} / 6$. Thus, equation (2) could be approximated by.

$$
\mathrm{v}_{\mathrm{o}} \square \mathrm{V}_{\mathrm{dc}}+\frac{2 \hat{\mathrm{V}}_{\mathrm{m}}}{\mathrm{m} \pi} \sin \left(\frac{\pi}{\mathrm{m}}\right) \cos (\mathrm{m} \omega \mathrm{t})
$$

The dominant ripple component in the output voltage is function in pulse numbers. Thus, as the pulse number increases, the ripple amplitude reduces significantly.

Moreover, the frequency of the ripple component is $\mathrm{m}$ times supply frequency. Therefore, the volumetric dimension of DC-filter reduces with the increase of pulse number.

The RMS value for the output voltage of MPDRs for series and parallel topologies is given by equation (6) [11, $12]$. 


$$
V_{\text {orms }}=\left\{\begin{array}{c}
\hat{\mathrm{V}}_{m} \sqrt{m / 6}\left(\frac{m}{2 \pi}\left(\frac{\pi}{m}+\frac{1}{2} \sin \left(\frac{2 \pi}{m}\right)\right)\right)^{-2} \text { Series } \\
\hat{\mathrm{V}}_{m}\left(\frac{m}{2 \pi}\left(\frac{\pi}{m}+\frac{1}{2} \sin \left(\frac{2 \pi}{m}\right)\right)\right)^{-2} \text { Parallel }
\end{array}\right.
$$

The variation of ripples in the output voltage with pulse number is shown in Figure 3. In Figure 3, the Ripple Factor (RF) is illustrated to express the ripple in the output voltage. RF is calculated from DC voltage equations (3) and RMS value equation (6)

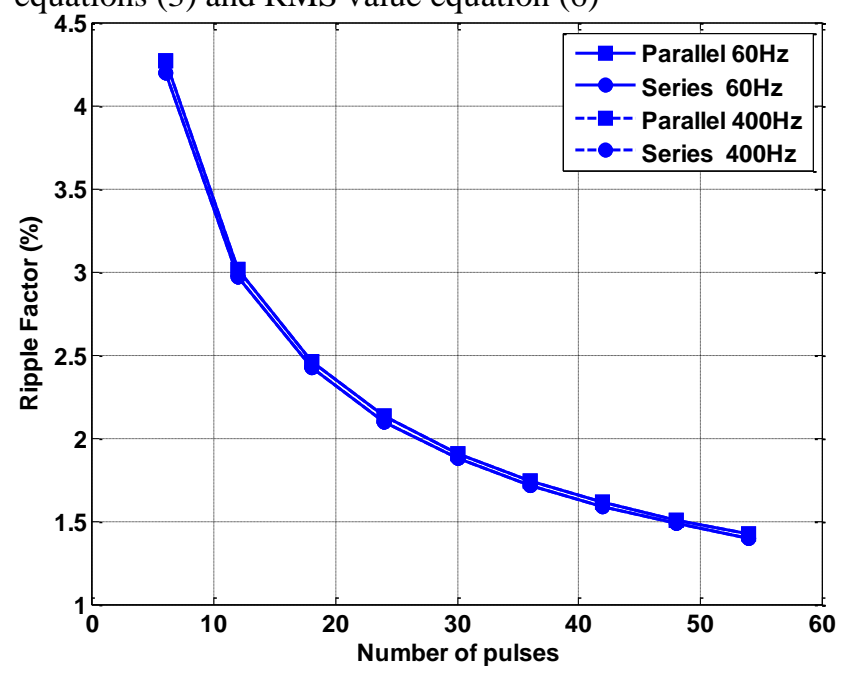

Figure 3: Amplitude of ripple in MPDRs output voltage versus pulse number for parallel (square) and series (circle), solid $(60 \mathrm{~Hz})$, dashed

$$
(400 \mathrm{~Hz})
$$

It is worth to mention that in producing Figure 3, the parameters of the diodes are those used in developing

$$
\begin{aligned}
& i_{o}=\mathrm{i}_{d c t r a n}+\mathrm{I}_{d c}+\mathrm{i}_{d c r}=\left(-\frac{\mathrm{V}_{\mathrm{dc}}}{R_{L}}-\sum_{n=m, 2 m}^{\infty} \frac{2 V_{m}}{\left(n^{2}-1\right)} \frac{m}{\pi} \sin \left(\frac{\pi}{m}\right) \cos \left(\frac{n \pi}{m}\right) \frac{n \omega L}{\sqrt{R_{L}^{2}+(n \omega L)^{2}}}\right) e^{--\frac{R_{L} t}{L}} \\
& +\frac{\mathrm{V}_{\mathrm{dc}}}{R_{L}}-\sum_{n=m, 2 m}^{\infty} \frac{2 V_{m}}{\left(n^{2}-1\right) \sqrt{R_{L}^{2}+(n \omega L)^{2}}} \frac{m}{\pi} \sin \left(\frac{\pi}{m}\right) \cos \left(\frac{n \pi}{m}\right) \cos \left(n \omega t-\theta_{n}\right)
\end{aligned}
$$

The conduction losses of MPDRs could be computed by,

$$
P_{c o n}=2\left(\frac{m}{6}\right)\left(I_{d R M S}^{2} R_{o n}+I_{d d c} V_{o n}\right)
$$

where $I_{d R M s}$ and $I_{d d c}$ are RMS and DC values of diode current respectively. Note that each diode in MPDRs conducts for $2 \pi / 3$ irrespective of arrangement either series or parallel. Thus, the diode dc current $\mathrm{I}_{\mathrm{ddc}}$ could be obtained from DC value of load current $\mathrm{I}_{\mathrm{dc}}$ by equation (9),
Figure 2. RF has highest of $4.2 \%$ at six-pulse operation. This value decreases rapidly as the number of pulse increases.

Series and parallel topologies are producing nearly the same level of ripples in output voltage, Figure 3. Both topologies could consider to have adequate performance regarding the output voltage, which is preliminary for applications requiring almost pure DC as batteries.

Figure 3 indicates either the topology type and/or supply frequency has almost negligible impact on the level of the ripple.

The load current $i_{o}$ could be calculated by equation (7) for R-L load. The current $i_{o}$ as shown in (7) is composed of three components, $\mathrm{i}_{\mathrm{dctran}}, \mathrm{I}_{\mathrm{dc}}$ and $\mathrm{i}_{\mathrm{dcss}}$.

The exponential components in (7) represents the transient component of load current $i_{\text {dctran }}$. This component arises post large transient as abrupt load change and they eventually decay.

The steady-state DC current component $\mathrm{I}_{\mathrm{dc}}$ accounts for the power transferred from the supply to the load. The current component $i_{\text {dcss }}$ represents the ripples in steadystate current. The amplitude of this component depends on pulse number and value of DC side filter $\mathrm{L}$. 
The conduction losses calculated by equation (8) are illustrated in Figure 4 for series and parallel arrangement of MPDRs. The base power for the conduction losses is taken $1 \mathrm{~kW}$, Figure 4 . The parameters of the diode in Figure 4 are those used in developing Figure 2.

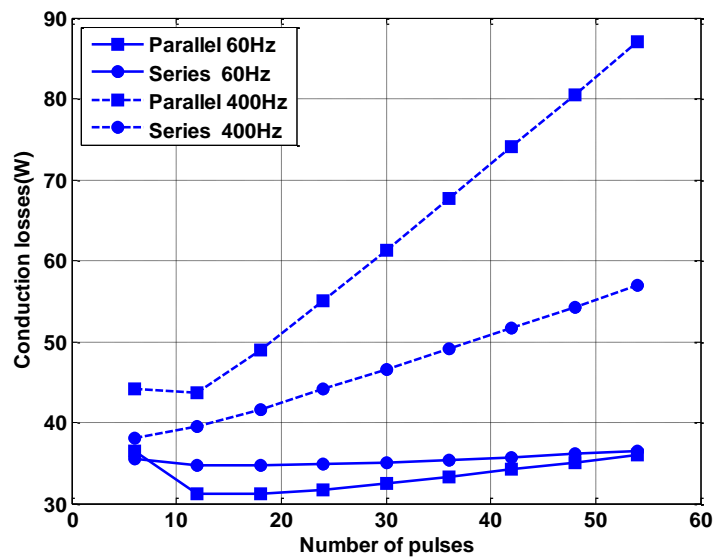

Figure 4 : Conduction losses for parallel and series arrangement for parallel (square) and series (circle), solid $(60 \mathrm{~Hz})$, dashed $(400 \mathrm{~Hz})$

In developing Figure 4, MPDRs, the load power is assumed constant at $10 \mathrm{~kW}$ irrespective to pulse number. Thus, the load average current in parallel topology will be higher than series arrangement particularly at high pulse number.

Series topology of MPDRs results in higher conduction losses than the parallel one at wide range of pulse number, Figure 4. Moreover, as pulse number increases the conduction losses of the series topology is reduced for constant load power operation. This is attributed to the high value of load DC voltage at high pulse number, which reduces DC currents and hence losses, (8).

Conduction losses of MPDRs are affected by supply frequency, they are increasing significantly for high frequency supplies such as aircraft, Figure 4. Moreover, parallel MPDRs offer higher conduction losses than series MPDRs for high frequency supplies.

It is composed of two-six pulse connected to secondary of three windings transformer. There is $30^{\circ}$ between the secondaries. Equation (11) is valid for series and parallel topologies.

Figure 5 illustrates Total Harmonic Distortion (THD) for primary current of MPDR at different pulse numbers. In developing Figure 5, the load is resistive $10 \mathrm{k} \Omega$ and no filtering arrangement is used in DC or AC sides.
Figure 4 shows that the conduction losses are insignificant for MPDRs, they account for around $0.4 \%$ of load power. This exposes the advantage of MPDRs in operating with extremely high efficiency, as they operate with zero switching losses.

It is worth to mention that the efficiency of MPDRs is also affected by reverse recovery losses. However, reverse recovery losses could be reduced by proper choice of the diode. Moreover, reverse recovery time has no significant for rectifier operation on $50 / 60 \mathrm{~Hz}$ supply.

\subsection{Input Side of MPDR}

The primary current of transformer interfacing MPDR to grid has multi-step nature. The number of steps per half cycle of the primary current depends on number of basic building block, six-pulse bridge, which are forming MPDR. For example, the primary input current has only one step of $120^{\circ}$ width per half cycle, when the MPDR is the basic six-pulse diode rectifier. However, for twelvepulse diode rectifier the current has two steps. Thus, the number of pulses per half cycle and increases with the pulse number. Moreover, the off-periods in the supply current are reducing also as the pulse number increases. Thus, MPDR with high pulse number would probably have less THD to such degree to comply with harmonic standards [11] without cumbersome harmonic filter. This is the key issue for producing MPDRs.

The primary current of MPDRs has the same shape irrespective for series or parallel operation for the same pulse number. The primary current of MPDR is sum of the currents of the transformer secondaries referred to the primary. The currents of the transformer secondaries have the phase shift equal to $\theta$ given by (1). For example for MPDR with $m=12$, the current of the supply phase a $i_{a}$ could be given by (11). This equation is obtained from the configuration of the twelve-pulse rectifier.

$i_{a}=\sum_{n=1,3,5}^{\infty} I_{d c}\left(\frac{4}{n \pi} \cos (n \alpha)+\frac{2}{n \pi} \sin \left(\frac{n \beta}{2}\right)\left(\sin \left(\frac{n \pi}{2}\right)-\sin \left(\frac{3 n \pi}{2}\right)\right)\right) \sin (n \omega t)$

(11)

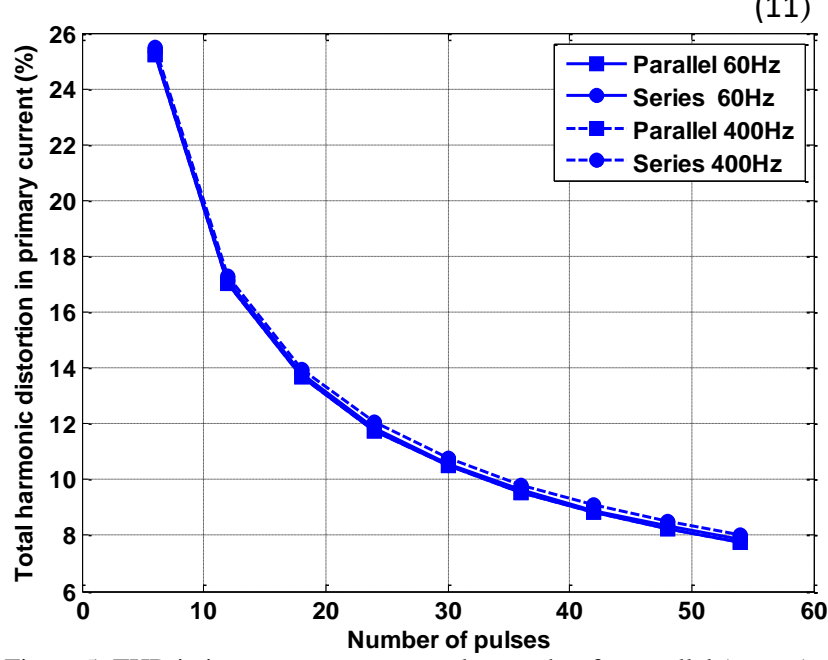

Figure 5: THD in input current versus pulse number for parallel (square) and series (circle), solid $(60 \mathrm{~Hz})$, dashed $(400 \mathrm{~Hz})$ 
Figure 5 shows that the THD in the primary current depends on number of factors:

1. Pulse number; number of six-step rectifier operating.

2. Load on the DC side

3. Filtering requirements in $\mathrm{DC}$ and $\mathrm{AC}$ sides.

Therefore, Figure 5 represents specific case as mention. It depicts THD for MPDRs in series and parallel arrangement for $10 \mathrm{k} \Omega$ without filtering facilities. Figure 5 indicates the MPDRs offer nearly the same THD irrespective to the topology and/or the frequency of the supply. Moreover, THD reduces with the pulse number. It is worth to mention that the THD in Figure 5 could be reduced even more via using appropriate filtering requirements.

\section{Experimental validation}

The test-rig used for the validation is composed of threephase transformer, two six-step bridge and load. A threephase, three windings transformer $500 \mathrm{VA}, 60 \mathrm{~Hz}$ is used in validation. The number of turns of the three windings respectively are 408, 214 and 214. The supply is threephase, $400 \mathrm{~V}$ and $60 \mathrm{~Hz}$. The transformer has the flexibility to be connected either star or delta in the three windings. However, there is no ability to increase/decrease the voltage. Diode bridge is implemented from six diode $600 \mathrm{~V}, 2 \mathrm{~A}$. The primary, secondary and tertiary are connected delta, delta and star. The output voltage for six-step and twelve-pulse series MPDRs are shown in Figures 6-8 respectively. Figure 7 shows the output voltage of twelve-pulse rectifier from the simulation

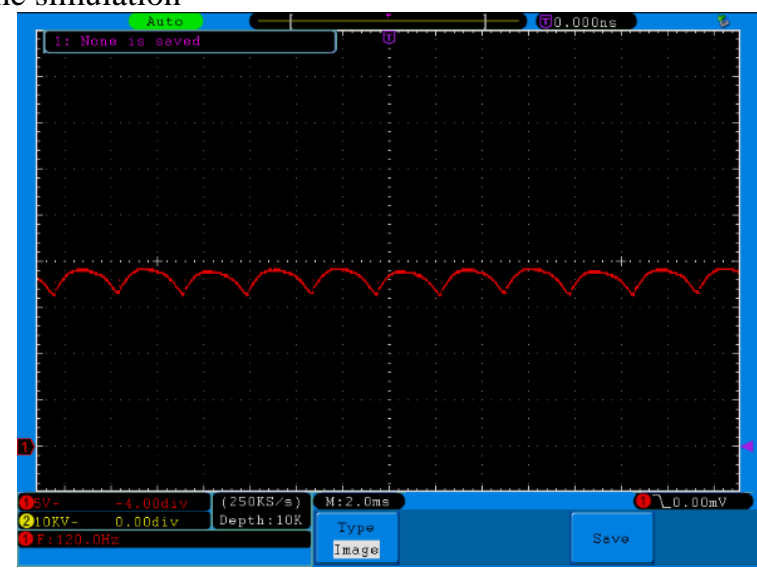

Figure 6: Output voltage of a six- pulse diode rectifier

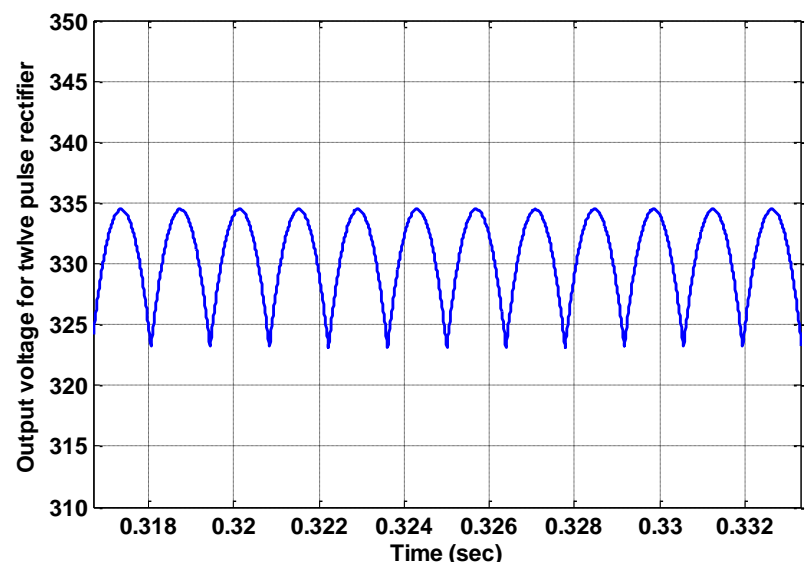

Figure 7 : Output voltage of a twelve-pulse diode rectifier from simulation (series connection)

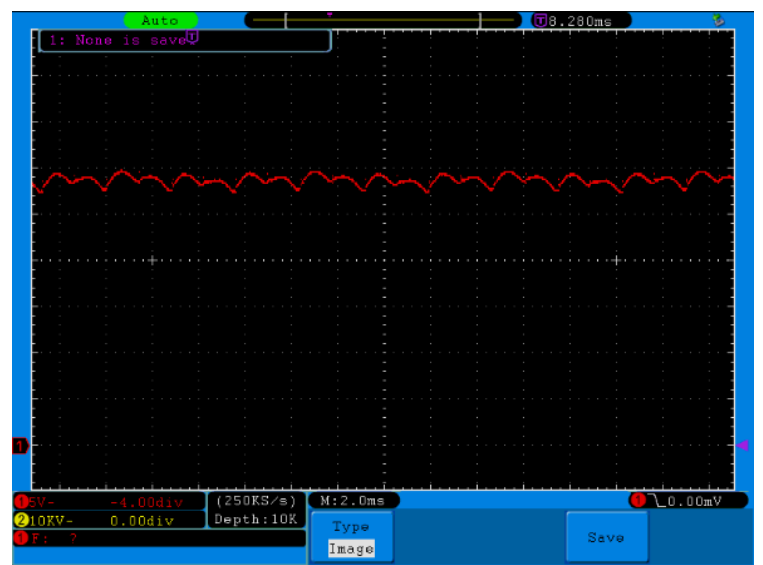

Figure 8 : Output voltage of a twelve-pulse diode rectifier from test-rig (series connection)

There is discrepancy between simulation and experimental results shown in Figures 7 and 8. This attributed to the difference between the output voltage level of the series connected bridges. As, one bridge is attached to the delta secondary, while the other is attached to the star secondary. The delta side is $57.7 \%$ less than star side. The output voltage for series MPDR is the sum of the output voltage of the two six-step diode rectifier.

The input current for six-step and twelve-pulse MPDRs are shown in Figures 9 and 10 respectively.

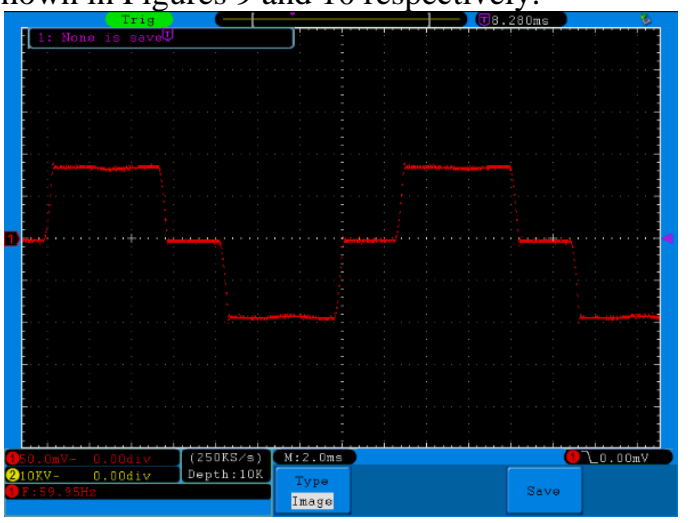

Figure 9: Primary current of six-pulse diode rectifier 


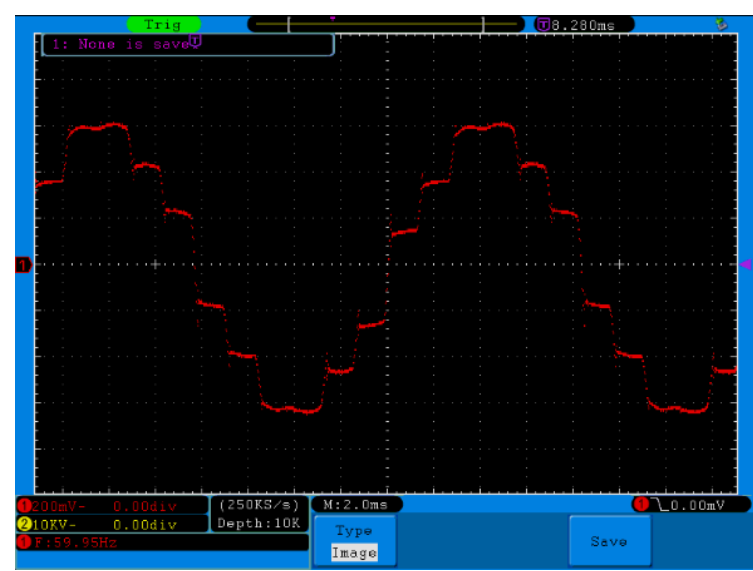

Figure 10 : Primary current of twelve-pulse diode rectifier (series connected)

It is difficult to realize parallel connection of twelve-pulse MPDR, due to the limitation of the transformer in the laboratory. As, mentioned, there is around $57.74 \%$ reduction in the voltage between the star and delta secondaries. Parallel connection of the DC side of the two six-pulse rectifier in such condition results in revering the bridge connected to delta secondary. Thus, the operation reverts from twelve-pulse to six-pulse.

A photo for the test-rig is shown in Figure 11.

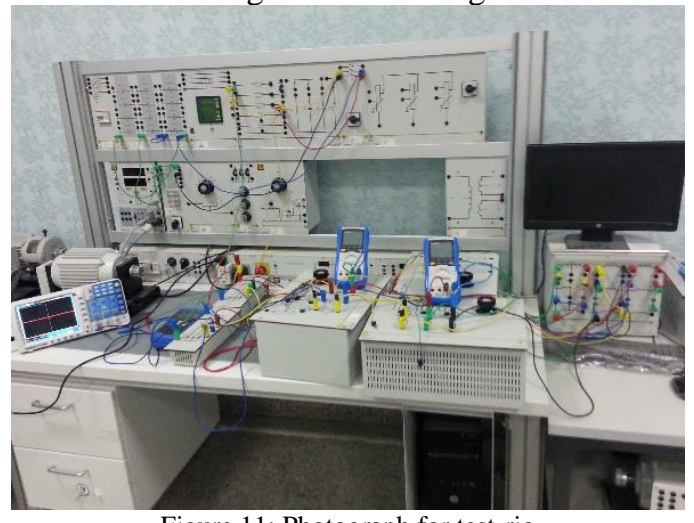

Figure 11: Photograph for test-rig

\section{Conclusions}

The series and parallel arrangements of MPDRs are thoroughly analyzed regarding the quality of current and voltage of DC and AC sides. Annotative analytical formulas are derived for different parameters of series and parallel topologies of MPDRs. A number of items could be considered as conclusion, such as :-

1. Series and parallel arrangements of MPDRs offer the same quality for input and output voltage and current. This is shown clearly in Figure 3 for RF in output voltage and Figure 5 for THD in input current.

2. Series arrangement of MPDRs enjoys the merit of providing high DC output. However, this for high power application demands diodes with high current rating, which increases the cost and complicates heat dissipation management.

3. Parallel-connected MPDRs have the merits of reduced current and voltage ratings. Moreover, their efficiency is significantly higher than their contender series topology.

4. Parallel topology of MPDRs are fault-tolerant. They could continue to function even a fault disabling one bridge or more depending on the number of pulses. However, the output power is reduced under such conditions. Moreover, THD is increased in the input current, which mandates fast remedy strategy to reduce the level of emitted harmonics.

5. Series arrangement of MPDRs lack fault-tolerance feature, as a fault disabling one diode results in deteriorating the performance of the whole topology irrespective number of pulses.

6. MPDRs suffer from the dependency of load voltage on load; thus for variable load operation, appropriate load voltage regulating approach has to be used.

\section{References}

[1] T. Glennon, " Fault tolerant generating and distribution system architecture," in IEE Colloquium on All Electric Aircraft, pp. 1-4, June 1998.

[2] M. Maldonado and G. Korba " Power management and distribution system for a more-electric aircraft (MADMEL)," IEEE Aerospace and Electronic Systems Magazine, vol. 14, pp. 38, 1999.

[3] S. Mollov, A. J. Forsyth and M. Bailey " System modelling of advanced electric power distribution architecture for large aircraft," SAE Transaction, pp. 904-913, 2000.

[4] Kaz Furmanczyk and Mark Stefanich, "Demonstration of very high power airborne AC to DC converter," in SAE Power System Conference, Reno, Nevada, Nov. 2-4, 2004.

[5] F.J.Chivite-Zabalza, A.J. Forsyth, D.R.Trainer, "Analysis and practical evaluation of an 18-pulse rectifier for aerospace applications," in Proceedings of the Second International Conference on Power Electronics, Machines and Drives,2004, vol 1. pp. 338-343.

[6] S. Choi; B. Sup Lee; and P. N. Enjeti " New 24-Pulse Diode Rectifier Systems for Utility Interface of High-Power AC Motor Drives" IEEE TRANSACTIONS ON INDUSTRY APPLICATIONS, Vol. 33, NO. 2, , pp. 531-541, 1997.

[7] S. Choi, P. N. Enjeti, and H. Lee, "A new reduced kVA multipulse diode rectifier front end for high power AC motor drives draws near sinusoidal input currents," in Proceedings of International Conference Power Electronics, pp. 110-115, Oct. 1995.

[8] S. Rosado; R. Burgos; F. Wang; and D. Boroyevich " Large- and Small-Signal Evaluation of Average Models for Multi-Pulse Diode Rectifiers" IEEE COMPEL Workshop, Rensselaer Polytechnic Institute, Troy, NY, USA, pp. 89-94, July 16-19, 2006.

[9] H. Zhu, R. P. Burgos, F. Lacaux, A. Uan-Zo-li, D.K. Lindner, F. Wang, D. Boroyevich, "Average modeling of three-phase and nine-phase diode rectifiers with improved AC current and DC voltage dynamics," Proceedings of IECON 2005. pp. 6-10, Nov. 2005 .

[10] IEEE-Std 519-1992, IEEE Recommended Practices and Requirements for Harmonic Control in Electrical Power Systems, IEEE Inc., New York, NY, April 1993. 
[11] M. H. Rashid " Power Electronics: Circuits, Devices \& Applications" 4th edition, Pearson; 2013.

[12] N. Mohan " Power electronics : converters, applications and design" 